\title{
Factor structure of the Indonesian version of the Parent as Social Context Questionnaire
}

\author{
Fitri Ariyanti Abidin ${ }^{1}$, Rismijati E. Koesma ${ }^{2}$, Poeti Joefiani ${ }^{3}$, Juke Roosjati Siregar ${ }^{4}$ \\ ${ }^{1,2,3,4}$ Faculty of Psychology, Universitas Padjadjaran, Indonesia \\ ${ }^{1}$ fitri.ariyanti.abidin@ unpad.ac.id., tettyris23@gmail.com., ${ }^{3}$ jpoeti@yahoo.com., ${ }^{4}$ jukesiregar@yahoo.co.id.
}

\section{ARTICLE INFO}

Article history

Received 4 February 2019

Revised 24 July 2019

Accepted 25 July 2019

Keywords

adolescent

parenting dimension

PSCQ

\begin{abstract}
The use of a dimensional approach in assessment tools of parenting style in Indonesia is still very limited. Parent as Social Context Questionnaire (PSCQ)-Adolescent Report is widely known as a dimensional-parenting style assessment tool. This study examined the factor structure and reliability of the Indonesian version of the PSCQ-Adolescent Report. This 24-item questionnaire measures six parenting dimensions: warmth, structure, autonomy support, rejection, chaos, and coercion, with four items in each dimension. Participants were 1476 Junior High School Students aged 11-16 years old ( $48 \%$ male, $52 \%$ female), recruited from six main islands in Indonesia. Our findings confirmed the original six unipolar factor structures of the Indonesian PSCQ. Of the six factors, five factors with the exception of coercion had acceptable to good reliability. The finding that coercion had no negative correlation with the positive dimensions such as warmth and autonomy support differs from findings in the West, indicating that coercion is considered negative in Western culture but is not the case in Indonesia. This study concluded that PSCQ-Adolescent Report can be used as a valid and reliable measure of the parenting style of Indonesian adolescents.
\end{abstract}

\section{Introduction}

Parents are significant others who have a strong influence on their children and remains to have so in the stages of early adolescence. The rapid change of physical, cognitive, social, and social context associated with adolescent development propose new issues and concern into the parent-child relationship (Branje, 2018). Studies on parenting adolescents are essential for two intertwined reasons. First, understanding the nature of parenting behavior can lead to positive outcomes of adolescent behavior, such as high well-being, self-esteem, and life satisfaction (Hoskins, 2014). Second, understanding can lead to an optimal intervention in enhancing the quality of parenting when the adolescents become parents themselves (Haggerty, McGlynn-Wright, \& Klima, 2013). To understand parents' influence on their children, there are three aspects of parenting that must be understood: the goals parents promote, how parents reach those goals or parenting practices, and emotional climate within the family or parenting style. Those three aspects of parenting are interconnected (Vermeer, 2011).

There are two major approaches in studying parenting style: typological approach and dimensional approach (Power, 2013). The typological approach to parenting usually combines specific dimensions of parenting to form parenting styles or clusters. Baumrind's parenting typologies (authoritative, authoritarian, and permissive type) have served as the 
template for many recent studies (Power, 2013), including research on parenting in Indonesia. A study in Indonesia was conducted using Baumrind's typological approach to examine the relationship between parenting style and other variables, e.g., a study on the effect of parenting style, peer group and self-efficacy on adolescent's sex behavior (Nurhidayah, Prestiana, \& Bayani, 2012). The typologies proposed by Baumrind is a constellation of two independent dimensions, namely demandingness and responsiveness. Demandingness refers to parents' readiness to confront a defiant child and ask for mature behavior and compliance with parental directives. Responsiveness refers to affective warmth and autonomy-support. An authoritative parenting style is a combination of highly demanding and highly responsive parents or sometimes labelled as democratic parenting. Authoritarian parenting is characterized as highly demanding but not responsive. In contrast, permissive parenting is a combination of highly responsive but non-demanding parenting (Baumrind, 2013). On the other hand, dimensional approach studies each of the dimensions of parenting independently - for example, the study of structure as the provision of clear and consistent rules, expectation, and consequences (Grolnick \& Pomerantz, 2009). In Indonesia, a study that used the dimensional approach was conducted with a focus on the warmth dimension (Fithria, 2010). From the best of our knowledge, the number of studies using this approach in Indonesia is relatively smaller than the number of research using a typological approach, specifically the typology of Baumrind.

Each approach has its strength and weaknesses (Power, 2013). This study used a dimensional approach for the following reasons: First, the isolated contribution of each parenting dimension facilitates a theoretical explanation for understanding the effect of parenting dimensions on any chosen dependent variable (Chew \& Wang, 2015). Second, because parenting is depicted as dimensional, cross-cultural researchers may be able to examine a particular dimension in a different culture, to see whether that construct exist universally (Tan, 2012).

Skinner, Johnson, \& Snyder (2005) formulated a comprehensive parenting study using the dimensional approach consist of six core dimensions: (1) warmth, (2) structure, (3) autonomy support, (4) rejection, (5) chaos, and (6) coercion. Warmth, structure, and autonomy support are labelled as positive dimensions. Emotional availability, support, and genuine caring as the expression of affection, love, appreciation, kindness, and regard represent warmth. Warmth is an essential dimension of parenting, salient in almost all conceptualizations of parenting. Several constructs related to warmth are approval, acceptance, love, support, supportive control, positive involvement, closeness, connection and child-centredness. Structure involves giving knowledge of the ways to achieve the expected goals, setting a reasonable limit and expectation.

According to Skinner et al. (2005), demandingness, firm control, behavioral control, contingent responsiveness, behavior contingency, direction, assertive control, strictness, supervision, organization, regulation, rule-setting, and regularity of routine are constructs that have been examined by other scholars in relation to the construct of "structure". Farkas and Grolnick (2010) used these constructs to develop a six-component conceptualization of structure. The six components are (1) Clear and consistent rules, guidelines, and expectations; this component is related to strictness and supervision. (2) Predictability which involves clear and consistent consequences of and contingencies for actions and consistent discipline. (3) Task-focused information feedback; this component is related to the positive feedback from the environment on children's meeting expectations. (4) Provision of opportunities to meet expectations by providing time, resources, and assistance. (5) The provision of rationales for rules and expectations; including "why explanation" to children. (6) Authority which involves behavioral control. Autonomy support is more than the chance given to children to express their thought and feeling regarding planning and problem solving. Other constructs related to autonomy 
support are psychological autonomy, freedom, responsiveness, democratic, non-directive and autonomy-granting.

Meanwhile, the next three dimensions are labeled as negative dimensions (Skinner et al., 2005). Rejection refers to hostility and expression of rejection when the parent actively dislikes his/her children. Hostility, irritability and criticism are some expressions of parental rejection. Chaos refers to parenting behaviors which do not give clarity to the child, including inconsistency and unpredictability.

Several constructs related to chaos are permissiveness, non-direction, lax control, unpredictability, laissez-faire, and inconsistent discipline. Coercion is a restrictive over controlling intrusive autocratic style which demands strict obedience. Another construct related to coercion is arbitrary control, psychological control, inflexibility, rigid discipline, intrusive control, strict control, and power assertion.

Based on this operationalization Skinner et al. (2005) developed a dimensional selfreport measurement tool named Parent as Social Context Questionnaire (PSCQ) that has been used in numerous studies (Farkas \& Grolnick, 2010; Hardy, White, Zhang, \& Ruchty, 2011; McLachlan, Zimmer-Gembeck, \& McGregor, 2010) The advantages of this measurement are its theoretical foundation, concise in measuring parenting dimensions, and ability to capture the core dimensions of parenting style (Skinner et al., 2005). There are two forms of the PSCQ; the PSCQ-Parent Report (to be filled in by the parent) and the PSCQ-Adolescent Report (to be filled in by the adolescent). In regards to past scorings of the dimensions, it has been common practice for the researcher to assess dimensions of parenting style as bipolar, such as warmth versus hostility. However, according to Skinner's study (Skinner et al., 2005), parenting is better assessed when dimensions of parenting style are evaluated as a separate construct (i.e., autonomy support and coercion) rather than bipolar dimensions (i.e., autonomy support versus coercion). Further studies which examined the factor structure of PSCQ-Adolescent Report supported Skinner's unidimensional model. Chew \& Wang (2015) compared the six-factor model against alternative three-factor model on secondary student-athletes in Singapore and found that six-factor unipolar model attained a significantly better fit to the data than the three-factor bipolar model in both maternal and paternal models. The factor structure of PSCQ was also studied in the Asian context among 11-13 years subjects using Rasch perspective (Tan, 2012) resulting in six dimensions, namely warmth, autonomy support, structure-control, structure-warmth, chaos, and negative parenting.

Research on parenting in the Indonesian context is still limited, relative to research on parenting in the Western context which has studied various approaches and its implications for child development (Riany, Meredith, \& Cuskelly, 2016). For researchers who use a quantitative approach in studying parenting in the Indonesian context, standardized measurement is essential. From the best of our knowledge, there is only one study that use PSCQ-Parent Report, with three bipolar models. In this model, autonomy support and coercion were treated as a bipolar dimension, namely parental autonomy support. Warmth and rejection were treated as a bipolar dimension called parental involvement, while structure and chaos were treated as a bipolar dimension called parental structure. The reliability of each dimensions were $\alpha=.593$ for parental autonomy support, $\alpha=.837$ for parental involvement, and $\alpha=.732$ for parental structure, while report of validity was unavailable (Tjioe \& Hildayani, 2012). Building on the limited study on measurement of parenting style using dimensional approach and due to the importance of adolescents' perception of themselves, the present study aimed to examine the factor structure and reliability of Indonesian version of the PSCQ-Adolescent Report. We used a six-factor model as a hypothetical model for this measurement. 


\section{Method}

\section{Participants}

Ethical clearance was obtained from the Ethical Committee of Padjadjaran University. Junior high school students $(n=1476)$ aged 11 to 16 years from 14 urban-areas in Java, Sumatera, Kalimantan, Sulawesi, Papua, and Maluku as a representation of the diverse ethnic groups in Indonesia were selected to participate in this study. Non-probability sampling was applied in selecting the participants based on the decision of the school authority. Since many rural areas still use ethnic languages to communicate, the choice of conducting the study in urban areas ensures that participants will understand the language used in the measurement (i.e., Bahasa Indonesia). School authority helped ensure that Bahasa Indonesia was the native language used in the participants' daily life.

\section{Procedure}

This study involved 14 research assistants who majored in Psychology. All research assistants received two online training as a group: one session was about the PSCQAdolescent Report and the other on data collection procedure. In any case where there were specific issues regarding a group of participants, the researchers provided individual follow-up training by phone, e.g. when the ethnicity in one group of participants did not available on the computerized answer sheet.

Next, the research assistants approached schools in his/her area to participate in the study. Approval from relevant school authorities and informed consent from the adolescents were then obtained. The class was selected by the school authority and is dependent upon certain situational factors (e.g., whether or not teachers give permission) as the data collection was done during school time. Then the questionnaires were administrated to and filled in by all students in each classroom. The completion of the questionnaire took approximately 30-45 minutes and used a computerized answer sheet. Finally, the answer sheets were sent to the first author of this article.

\section{Instruments}

The 24-item PSCQ-Adolescent Report (Skinner et al., 2005) was used to measure the six parenting dimensions: (1) warmth, (2) rejection, (3) structure, (4) chaos, (5) autonomy support, (6) coercion. Each of the six dimensions contains four 4-point Likert scales $(1=$ not at all true to $4=$ very true). After getting permission from the author of the PSCQAdolescent Report, the English version was then translated to Bahasa Indonesia and then translated back to English. Translation was done by two translators who were required to have majored in psychology, have achieved a minimum of 550 on their TOEFL (Test of English as Foreign Language) scores, and had experience in translating documents in academic contexts. As different translations were found in several words, a panel discussion was conducted between the researcher and the translators to determine the choice of words that best fit the concept. While in the original version, "my parents" is used in the instruction of the questionnaire, in our study a list of possible caregiver figures was given (such as mother, father, grandmother, stepmother, sister/brother, other relatives) to accommodate participants who were not living with their parents. Thus the participants were able to choose the caregiver that fitted their situation.

\section{Data Analysis}

Confirmatory Factor Analysis (CFA) with the Lisrel Software Version 9.30 was used to estimate the composite reliability and factor structure of the PSCQ-Adolescent Report. A minimum of .70 was expected as an indication of good reliability (Hair Jr, William, Babin, 
\& Anderson, 2014). First, we calculated the CFA for each dimension of the PSCQAdolescent Report. Then, we calculated the CFA for PSCQ-Adolescent Report to test the hypothesis of the six-factor model. Multiple criteria were used to assess the model's goodness of fit; including GFI as an absolute fit index, NFI as an incremental fit index, CFI as a goodness-of-fit index, and RMSEA as a badness-of-fit index. To be considered as a model fit, the values of GFI, NFI, and CFI should be at least .90, while the RMSEA value should be less than .08 (Hair Jr et al., 2014). We also evaluated the standardized loading estimates (factor loadings) of each item. The expected value of factor loadings is greater than .40 with a significant $t$ statistic (Wang, Andrade, \& Gorenstein, 2005).

\section{Results}

The participants of this study came from various ethnicities and religions, with parents who had different educational levels. Table 1 shows the proportion of participants from Java was $54.7 \%$, followed by Sumatera (22\%), Papua (7.9\%), Kalimantan (7.5\%), Sulawesi $(4.3 \%)$ and Maluku (3.7\%). There was a slightly higher percentage of female than male (52\% versus $48 \%)$, with most participants being 13 and 14 years of age $(M=13.4$; $\mathrm{SD}=1.23)$.

Table 1

Demographics of Participants

\begin{tabular}{|c|c|c|c|c|c|c|}
\hline \multirow{2}{*}{ Variables } & \multicolumn{6}{|c|}{ Number of Participants (\%) } \\
\hline & Java & Sumatera & Kalimantan & Sulawesi & Maluku & Papua \\
\hline Total Participants & 808 & 324 & 110 & 64 & 54 & 116 \\
\hline \multicolumn{7}{|l|}{ Gender } \\
\hline Male & $389(48.1)$ & $139(42.9)$ & $54(49.1)$ & $43(67.2)$ & $26(48.1)$ & $59(50.9)$ \\
\hline Female & $419(51.9)$ & $185(57.1)$ & $56(50.9)$ & $21(32.8)$ & $28(51.9)$ & $57(49.1)$ \\
\hline \multicolumn{7}{|l|}{ Ethnics } \\
\hline Java & $309(38.2)$ & $24(7.4)$ & $31(28.2)$ & $3(4.7)$ & - & $37(31.9)$ \\
\hline Sunda & $262(32.4)$ & $5(1.5)$ & $3(2.7)$ & - & - & $3(2.6)$ \\
\hline Batak & $9(1.1)$ & 99 (30.6) & $2(1.8)$ & - & - & - \\
\hline Bugis & $2(.2)$ & - & $2(1.8)$ & $46(71.9)$ & - & $55(47.4)$ \\
\hline Malay & $6(.7)$ & $36(11.1)$ & - & - & - & $2(1.7)$ \\
\hline Betawi & $94(11.6)$ & - & $1(.9)$ & - & - & - \\
\hline Banjar & - & - & $64(58.2)$ & & - & - \\
\hline Tionghoa & $60(7.4)$ & - & - & $1(1.6)$ & - & - \\
\hline Others & $66(8.2)$ & $160(49.4)$ & $7(6.4)$ & $14(21.9)$ & $54(100)$ & $19(16.4)$ \\
\hline \multicolumn{7}{|l|}{ Religion } \\
\hline Islam & $682(84.4)$ & $230(71)$ & $110(100)$ & $62(96.8)$ & $5(9.3)$ & $116(100)$ \\
\hline Christianity & $105(13)$ & $91(28.1)$ & - & $1(1.6)$ & 49 (90.7) & - \\
\hline Others & $21(2.6)$ & $3(\quad .9)$ & - & $1(1.6)$ & - & - \\
\hline
\end{tabular}

\section{Descriptive Statistics}

The mean of the positive dimensions is higher than that of the negative dimensions (see Table 2). This trend applies in the calculation of all participants as one whole as well as per island. In all participants, the warmth dimension had the highest mean (3.43), and rejection has the lowest mean (1.99). The same pattern was found for participants in Java, Sumatera, Kalimantan, and Papua. In Sulawesi, structure has the highest mean, while rejection has the lowest mean. Rather contrasting to the other islands, the highest mean in Maluku belongs to autonomy support, and the lowest is chaos. 
Table 2

Mean (M) and Standard Deviations (SD) of the PSCQ-Adolescent Report

\begin{tabular}{lccccccc}
\hline \multicolumn{1}{c}{ Sub Scale } & All & Java & Sumatera & Kalimantan & Sulawesi & Maluku & Papua \\
\hline & M (SD) & M (SD) & M (SD) & M (SD) & M (SD) & M (SD) & M (SD) \\
Warmth & $3.43(.52)$ & $3.48(.47)$ & $3.38(.62)$ & $3.40(.48)$ & $3.30(.57)$ & $3.30(.57)$ & $3.36(.52)$ \\
Structure & $3.28(.51)$ & $3.29(.47)$ & $3.27(.60)$ & $3.29(.53)$ & $3.40(.56)$ & $3.17(.56)$ & $3.18(.51)$ \\
Autonomy & $3.34(.46)$ & $3.36(.43)$ & $3.26(.53)$ & $3.38(.41)$ & $3.35(.43)$ & $3.35(.43)$ & $3.31(.42)$ \\
Support & & & & & & & \\
Rejection & $1.99(.57)$ & $1.97(.55)$ & $1.97(.61)$ & $1.93(.55)$ & $2.07(.69)$ & $2.15(.62)$ & $2.11(.59)$ \\
Chaos & $2.31(.56)$ & $2.30(.57)$ & $2.28(.58)$ & $2.39(.49)$ & $2.47(.58)$ & $2.11(.53)$ & $2.40(.53)$ \\
Coercion & $2.40(.56)$ & $2.38(.56)$ & $2.39(.58)$ & $2.43(.57)$ & $2.49(.59)$ & $2.53(.51)$ & $2.51(.52)$ \\
\hline
\end{tabular}

As expected, the positive dimensions (warmth, structure, autonomy support) are positively intercorrelated (with correlation coefficients ranging from .54 to .61, see Table 3). Likewise, the negative dimensions (rejection, chaos, coercion) are positive intercorrelated (with correlation coefficients ranging from .40 to .50). Rejection and chaos as negative dimensions are slightly negatively correlated with all positive dimensions (from -.07 to -.26). Meanwhile, coercion has no significant correlation with warmth and autonomy support and only a low negative correlation with structure.

Table 3

Intercorrelations between Dimensions of the Indonesian Version of PSCQ-Adolescent Report

\begin{tabular}{llcccccc}
\hline & & 1 & 2 & 3 & 4 & 5 & 6 \\
\hline 1. & Warmth & - & $.55^{*}$ & $.61^{*}$ & $-.26^{*}$ & $-.17^{*}$ & .02 \\
2. & Structure & - & - & $.54^{*}$ & $-.15^{*}$ & $-.07^{*}$ & $-.08^{*}$ \\
3. $\quad$ Autonomy Support & - & - & - & $-.24^{*}$ & $-.12^{*}$ & -.05 \\
4. Rejection & - & - & - & - & $.50^{*}$ & $.40^{*}$ \\
$5 . \quad$ Chaos & - & - & - & - & - & $.42^{*}$ \\
$6 . \quad$ Coercion & - & - & - & - & - & - \\
\hline$* . p<.05(2$ tailed $)$ & & & & &
\end{tabular}

\section{Reliability}

All dimensions had a sufficient composite reliability, except for coercion which fell slightly under the criterion of .70 (warmth $=.83$; structure $=.79$; autonomy support $=.70$; rejection $=.80$; chaos $=.74$; coercion $=.66$ ).

\section{Validity evidence based on the internal structure}

As shown in Table 4, each item achieved a sufficient standardized factor loading, except for one item in the coercion dimension (.37). The CFA analysis confirmed that each of the dimension has a good fit model. 
Table 4

The Goodness of Fit Indices of Each Dimension of PSCQ-Adolescent Report

\begin{tabular}{lccccccc}
\hline Dimensions & Range of FL & Chi-Square & Df & RMSEA & GFI & CFI & NFI \\
\hline Warmth & $.58-.72$ & .33 & 2 & .000 & .99 & .99 & .99 \\
Structure & $.52-.67$ & 12.44 & 2 & .061 & .99 & .99 & .99 \\
Autonomy & $.49-.56$ & 5.46 & 2 & .035 & .99 & .99 & .99 \\
Support & & & & & & & \\
Rejection & $.58-.66$ & 18.39 & 2 & .076 & .99 & .99 & .99 \\
Chaos & $.49-.61$ & 19.14 & 2 & .078 & .99 & .98 & .98 \\
Coercion & $.37-.59$ & 14.15 & 2 & .066 & .99 & .98 & .97 \\
\hline
\end{tabular}

The CFA calculation for six-factor model showed evidence of the good of fit criteria (Chi-Square $=671.99$ with df $=216$, RMSEA $=.039, \mathrm{GFI}=.96, \mathrm{CFI}=.97$ and NFI=96). The measurement model for PSCQ-Adolescent Report Indonesian version can be seen in Figure 1.

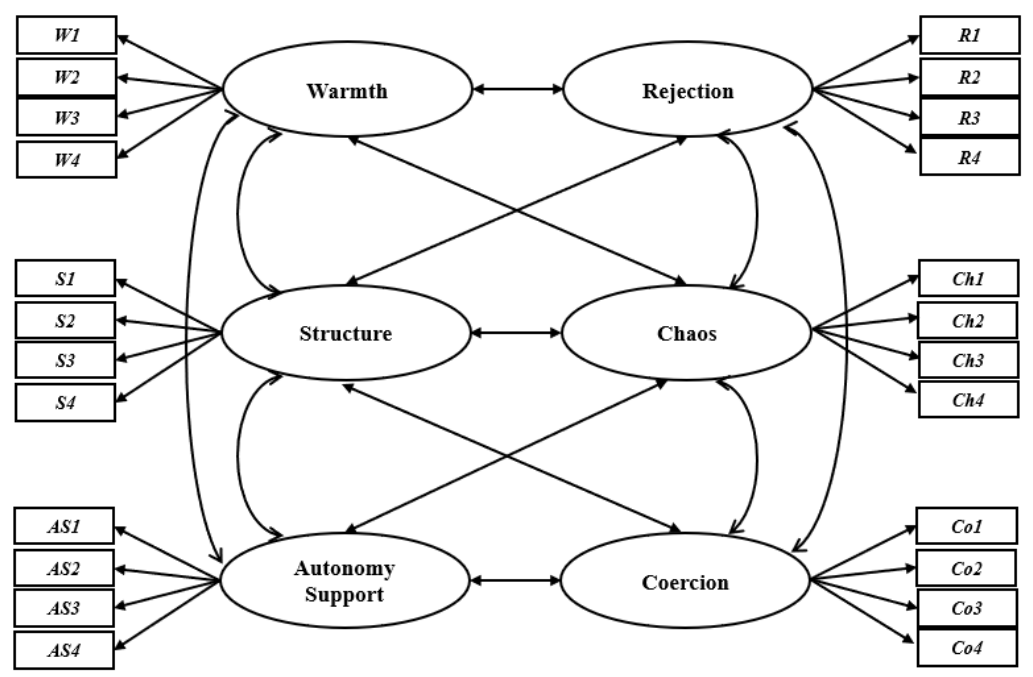

Figure 1. Model of Six Unipolar Factors for the Indonesian version of PSCQ-Adolescent Report

\section{Discussion}

The six unipolar models of PSCQ-Adolescent Report among Indonesian samples was identified using confirmatory factor analysis, confirming that the internal structure of the PSCQ-adolescent report consists of six unidimensional factors. This result is in line with other studies which investigated the factor structure of PSCQ-Adolescent Report (Chew \& Wang, 2015; Skinner et al., 2005) This result implies that each dimension in this measurement is unipolar. This finding is contrary to the assumption that parenting dimensions are bipolar (i.e., warmth vs. rejection, structure vs. chaos, autonomy support vs. coercion). It means that a higher score in the warmth dimension is not always followed by a lower score in the rejection dimension. This discovery also applies to structure and chaos, as well as to autonomy support and coercion. Another implication of the six unipolar constructs is that the researchers should not treat the negative dimensions as a negative counterpart of the positive dimensions. Subsequently, the researcher should not 
aggregate the scores of warmth and rejection, structure and chaos, or autonomy support and coercion. Evidence from this study suggests that when an intervention is applied, each dimension should be enhanced separately. In other words, a single intervention to enhance parents' warmth will not automatically decrease parents' rejection of their children.

The finding that coercion had no negative correlation with positive dimensions such as warmth and autonomy support differs from findings in the West. In the study of Skinner et al. (2005), coercion had significant negative correlations with all the positive dimensions (-.48 with warmth, -.46 with structure, and -.58 with autonomy support). This contrasting result and lower composite reliability for coercion compared to the other dimensions can be explained by cultural differences between the West and the East. Since parenting is influenced by culture (Bornstein, 2012), the same parenting construct may be seen differently between one culture to another. In Western culture, coercion is seen as a negative parenting dimension in which parents are responsible for the negative outcomes of their children (Paolo, Bornstein, Haynes, Rossi, \& Venuti, 2012). This conceptualization is based on the parenting goals of parents from Western cultures that is to produce independent, socially assertive, confident, and competent children (Rubin, Fredstrom, \& Bowker, 2008).

Meanwhile, in Eastern culture, especially in Asia, parenting is characterized by highly demanding and directive parents (Chao, 2009). Hierarchical social culture becomes a feature of South East Asian Countries, which requires younger persons to respect their elders and to view elders as superior to themselves. Indonesian parenting value also views that children have to follow their parents' guidance - no questions asked. Since the categorization of "positive" or "negative" parenting dimensions were based on the effect of that dimension on children's outcome. In contrast to western research findings, research in Asian adolescents found that control does not always have a negative impact on child outcomes because children perceive parental control as a way to show concern (Chao \& Aque, 2009).

Among the positive dimensions, warmth achieved the highest mean score (calculated by averaging the score of all participants), followed by autonomy support and structure. This result was in line with (Skinner et al., 2005) that showed a similar trend: warmth, autonomy support and structure. The structure as the lowest on the mean score compared with the other two positive dimensions also found in a study among Singapore adolescence (Chew \& Wang, 2015). The order of the mean score among the negative dimensions in this study is also in line with (Skinner et al., 2005). Interestingly, several unique findings were identified from participants of certain islands. For example, autonomy support was the highest mean score and chaos was the lowest mean score of participants from Maluku. This uniqueness requires cultural investigation, given that culture shapes parenting style (Bornstein, 2012).

In terms of limitations, while the present study involved a large number of Indonesian adolescents from different areas and major ethnic groups, we used convenience sampling to gather the data. Our study did not provide any information on adolescents in the rural areas, and might, therefore, not be generalizable to the adolescent population in Indonesia as a whole. Further, we restricted ourselves to validity and reliability. Consequently, we recommend future research to study a more representative group in order to obtain norms. Also, the test-retest reliability and construct validity of Indonesian version the PSCQ-Adolescent Report should be investigated as well, for example, by examining the relationship between dimensions of the PSCQ with other parenting measures that assess similar constructs. Finally, future studies should also explore the coercion construct, including possible cross-cultural differences in the items. It will also be interesting to discover the possibility of a different kind of coercion in the Indonesian context as well as its effect on the children's outcomes. 


\section{Conclusion}

The Indonesian version of the PSCQ-Adolescent Report is a valid and reliable measurement tool to asses parenting style using a dimensional approach. The dimensions in PSCQ-Adolescent Report are better to be used as a whole, even though each dimension need to be interpretated in their respective meaning (unipolar). Positive dimensions can be aggregated to form the positive parenting style. Further study is needed to get deeper understanding of coercion as one of the negative dimensions in the Indonesian context.

\section{Funding}

This study was funded by the Indonesian Endowment Fund for Education/ Lembaga Pengelola Dana Pendidikan (LPDP) RI through a scholarship for Fitri Ariyanti Abidin to attend her Doctorate at Faculty of Psychology Padjadjaran University.

\section{References}

Baumrind, D. (2013). Is a pejorative view of power assertion in the socialization process justified? Review of General Psychology, 17(4), 420-427. https://doi.org/10.1037/a0033480

Bean, R. A., Barber, B. K., \& Crane, D. R. (2006). Parental support, behavioral control, and psychological control among African American youth. Journal of Family Issues, 27(10), 1335-1355. https://doi.org/10.1177/0192513X06289649

Bornstein, M. H. (2012). Cultural approaches to parenting. Parenting, Science and Practice, 12(2-3), 212-221. https://doi.org/10.1080/15295192.2012.683359

Branje, S. (2018). Development of parent-adolescent relationships: Conflict interactions as a mechanism of change. Child Development Perspectives, 12(3), 171-176. https://doi.org/10.1111/cdep.12278

Chao, R. K. (1994). Beyond parental control and authoritarian parenting style: Understanding Chinese parenting through the cultural notion of training. Child Development, 65(4), 1111-1119. https://doi.org/10.1111/j.1467-8624.1994.tb00806.x

Chao, R. K., \& Aque, C. (2009). Interpretations of parental control by Asian immigrant and European American youth. Journal of Family Psychology, 23(3), 342-354. https://doi.org/10.1037/a0015828

Chew, W. C. E., \& Wang, C. K. J. (2015). Parents as social context in youth sport: A validation of the PASCQ with adolescent Singapore athletes PASCQ with adolescent Singapore athletes. National Institute of Education, (June), 1-15. https://doi.org/10.1017/jtp.2014.10.

Farkas, M. S., \& Grolnick, W. S. (2010). Examining the components and concomitants of parental structure in the academic domain. Motivation and Emotion, 34(3), 266-279. https://doi.org/10.1007/s11031-010-9176-7

Fithria. (2010). Family warmth dimension of bearing. Idea Nursing Journal, 1 No.1, 2126.

Grolnick, W. S., \& Pomerantz, E. M. (2009). Issues and challenges in studying parental control: Toward a new conceptualization. Child Development Perspectives, 3(3), 165170. https://doi.org/10.1111/j.1750-8606.2009.00099.x

Haggerty, K., McGlynn-Wright, A., \& Klima, T. (2013). Promising parenting programs for reducing adolescent problem behaviors. Journal Child Service, 8(4). 
https://doi.org/10.1108/JCS-04-2013-0016

Hair Jr, J. F., William, C., Babin, B. J., \& Anderson, R. E. (2014). Multivariate data analysis (Seventh Ed). Harlow: Pearson.

Hardy, S. A., White, J. A., Zhang, Z., \& Ruchty, J. (2011). Parenting and the socialization of religiousness and spirituality. Psychology of Religion and Spirituality, 3(3), 217230. https://doi.org/10.1037/a0021600

Hoskins, D. (2014). Consequences of parenting on adolescent outcomes. Societies, 4(3), 506-531. https://doi.org/10.3390/soc4030506

McLachlan, J., Zimmer-Gembeck, M. J., \& McGregor, L. (2010). Rejection sensitivity in childhood and early adolescence: Peer rejection and protective effects of parents and friends. Journal of Relationships Research, 1(1), 31-40. https://doi.org/10.1375/jrr.1.1.31

Nurhidayah, S., Prestiana, N. D. I., \& Bayani, I. (2012). Pengasuhan, peer group, self efficacy dan perilaku seks pada remaja di Kota Bekasi (Parenting, peer group, self efficacy and sexual behavior among adolescents in Bekasi City). Jurnal Soul, 5(2), $67-79$.

Paolo, V., Bornstein, M. H., Haynes, O. M., Rossi, G., \& Venuti, P. (2012). Infant behavior and development: A cross-cultural comparison of mothers' beliefs about their parenting very young children. Infant Behavior and Development, 35(3), 479488. https://doi.org/10.1016/j.infbeh.2012.02.006

Power, T. G. (2013). Parenting dimensions and styles: A brief history and recommendations for future research. Childhood Obesity, 9(s1), S-14-S-21. https://doi.org/10.1089/chi.2013.0034

Riany, Y. E., Meredith, P., \& Cuskelly, M. (2016). Understanding the influence of traditional cultural values on Indonesian parenting. Marriage \& Family Review, 4929(March). https://doi.org/10.1080/01494929.2016.1157561

Rubin, K., Fredstrom, B., \& Bowker, J. (2008). Future directions in friendship in childhood and early adolescence. Social Development, 17(4), 1085-1096. https://doi.org/10.1111/j.1467-9507.2007.00445.x

Skinner, E., Johnson, S., \& Snyder, T. (2005). Six dimensions of parenting: A motivational model. Parenting: Science and Practice, 5(2), 175-235. https://doi.org/10.1207/s153279220502

Tan, C. Y. (2012). Understanding Asian parenting from a Rasch perspective. Asian Journal of Social Psychology, 15(4), 273-283. https://doi.org/10.1111/j.1467839X.2012.01383.X

Tjioe, I. N., \& Hildayani, R. (2012). Pengaruh Parental Autonomy Support , Parental Involvement, dan Parental Structure terhadap Domain Kemandirian pada Remaja Penyandang Sindroma Down (The effect of parental autonomy support, parental involvement, and parental structure on Independence among Adolescents with Down Syndrom). (Master Thesis). Universitas Indonesia, Jakarta.

Vermeer, P. (2011). The impact of parental religiosity on parenting goals and parenting style: A Dutch perspective. Journal of Beliefs and Values, 32(1), 69-84. https://doi.org/10.1080/13617672.2011.549311

Wang, Y. P., Andrade, L. H., \& Gorenstein, C. (2005). Validation of the Beck Depression Inventory for a Portuguese-speaking Chinese community in Brazil. Brazilian Journal of Medical and Biological Research, 38(3), 399-408. https://doi.org/10.1590/S0100879X2005000300011 